\title{
Über einige Derivate des Brenzeateehins
}

\author{
von
}

\section{Oscar Wisinger.}

Aus dem I. chemischen Laboratorium der k. k. Universität in Wien.

(Vorgelegt in der Sitzung am 5. Juli 1900.)

Angesichts desUmstandes, dass die physiologischeWirkung mancher Arzneimittel durch den Eintritt einer Methoxyl- oder Äthoxylgruppe oder eine Alkylgruppe allein in mannigfacher Weise variiert wird, schien es von großem Interesse, derartige Substitutionsproducte des Phenacetins zu studieren und einen Weg zur Darstellung derselben zu finden. Über die zu diesem Ende führenden Versuche sei im nachstehenden berichtet, obwohl die physiologische Untersuchung am Thiere kein befriedigendes Resultat lieferte.

Als Ausgangsmaterial gelangte das käufliche Guajacol und Guätho1 zur Verwendung, und wurde zunächst aus dem ersteren der Methyläthyläther des Brenzcatechins gewonnen. Derselbe ist nach Tiemann und Koppe ${ }^{1}$ folgendermaßen dargestellt worden:

1 Molecül Guajacol wurde in äthylalkoholischer Lösung mit 1 Molecül Kalihydrat versetzt und durch 6 Stunden mit etwas mehr als 1 Molecül Jodäthyl am Rückflusse digeriert. Nach dem Abdestillieren des Alkohols wurde der Rückstand mit Wasser bis zur Lösung des gebildeten Jodkalis verdünnt, mit Äther ausgeschüttelt und schließlich eventuell unverändert gebliebenes Guajacol durch alkoholisches Kali entfernt. Das beim Abdunsten des Äthers zurückbleibende Ö1 wird im Vacuum getrocknet und destilliert. Übereinstimmend mit den Angaben

1 B., XIX, 2017. 
von Tiemann und Koppe wurde es als farblose, stark lichtbrechende, aromatisch riechende Flüssigkeit vom Siedepunkte $213^{\circ}$ erhalten. Aus $50 \mathrm{~g}$ Guajacol wurden $59 \mathrm{~g}$ Methyläthyläther erhalten, d. i. $96 \%$ der theoretischen Ausbeute.

Das Diäthylbrenzcatechin ist bisher nur von Kölle ${ }^{1}$ durch Destillation von Diäthylprotocatechusäure mit Kalk und von Herzig und $Z$ eisel ${ }^{2}$ durch Alkylierung von Brenzcatechin dargestellt worden. Letztere Autoren haben den Äther sehr schön krystallisiert (Schmelzpunkt 43 bis $45^{\circ}$ ) erhalten, während Kölle ihn als flüssig beschreibt.

In unserem Falle wurde der Diäthyläther aus dem neuestens technisch gewonnenen Äthyläther des. Brenzcatechins, dem Guäthol, dargestellt. Die Alkylierung wurde genau nach der beim Methyläthyläther angewendeten Methode durchgeführt, und zeigte der Äther ebenfails schöne Krystallisation und den von Herzig und Zeisel gefundenen Schmelzpunkt. Aus $50 \mathrm{~g}$ Guäthol wurden $57 \mathrm{~g}$ Diäthyläther gewonnen, d. i. $95 \%$ der theoretischen Ausbeute.

\section{Mononitrobrenzcatechindiäthyläther.}

Zur Darstellung des Mononitroproductes des Diäthyläthers wurde die Nitrierung in Eisessigverdünnung als passendster Weg gewählt. Und zwar wurden $50 \mathrm{~g}$ des sorgfältig getrockneten Äthers mit der vier- bis fünffachen Menge Eisessig versetzt und die zur Einführung einer Nitrogruppe ausreichende Menge 75-procentiger Salpetersäure, mit der drei- bis vierfachen Menge Eisessig verdünnt, unter Umschütteln zugesetzt. Der Eintritt der Reaction ist an der heftigen Erwärmung und dem Auftreten einer tief dunkelrothen Färbung kenntlich. Man beobachtet nun an möglichst rasch in Wasser gegossenen Proben der Mischung, ob eine ölige oder krystallinische Fällung entsteht. Im ersteren Falle ist noch einige Secunden unter Umschütteln zuzuwarten, im letzteren Falle sofort der ganze Inhalt des Kolbens unter Umrühren in ein mit 4 bis $5 l$ Wasser gefülltes Becherglas zu gießen. Bei zu langem Warten läuft

\footnotetext{
1 A., $159,246$.

2 Monatshefte für Chemie, X, 152.
} 
man Gefahr, dass das Product verharzt; bei zu frühem Eingießen hingegen, dass eine ölige Ausscheidung erfolgt. Hat sich der krystallinische Niederschlag zu großen Flocken zusammengeballt und die Flüssigkeit geklärt, so saugt man ab und krystallisiert das Rohproduct aus 95-procentigem Alkohol um. Der Nitrokörper scheidet sich in schönen hellgelben Nadeln $\mathrm{ab}$, die nach wiederholtem Umkrystallisieren den constanten Schmelzpunkt 73 bis $75^{\circ}$ besaßen.

I. $0.2073 \mathrm{~g}$ im Vacuum über Schwefelsäure getrocknete Substanz gaben $0 \cdot 4348 \mathrm{~g}$ Kohlensäure und $0 \cdot 1147 \mathrm{~g}$ Wasser.

II. $0 \cdot 2002 g$ im Vacuum über Schwefelsäure getrocknete Substanz gaben $0 \cdot 4168 \mathrm{~g}$ Kohlensäure und $0 \cdot 1122 \mathrm{~g}$ Wasser.

In 100 Theilen:

\begin{tabular}{|c|c|c|c|}
\hline & \multicolumn{2}{|c|}{ Gefunden } & \multirow{2}{*}{$\begin{array}{c}\text { Berechnet für } \\
\mathrm{C}_{6} \mathrm{H}_{3} \cdot \mathrm{NO}_{2} \cdot\left(\mathrm{OC}_{2} \mathrm{H}_{5}\right)_{2}\end{array}$} \\
\hline & I & II & \\
\hline & $.57 \cdot 2$ & $56 \cdot 78$ & $56 \cdot 87$ \\
\hline & $6 \cdot 15$ & $6 \cdot 22$ & $6 \cdot 16$ \\
\hline
\end{tabular}

$50 g$ Diäthyläther gaben $59 g$ Nitrokörper, d. i. $92 \%$ der theoretischen Ausbeute.

Während diese Arbeit im Gange war, wurden die Versuche von Cousin ${ }^{1}$ über nitrierte Guajacole publiciert, wobei ein Mononitroguajacol von unbekannter Stellung dargestellt wurde.

\section{$\alpha$ - und $\beta$-Mononitrobrenzcatechinmethyläthyläther.}

Die Nitrierung des Methyläthyläthers wurde auf genau gleiche Weise ausgeführt, und die Reaction zeigte denselben Verlauf. Nur beobachtete man in diesem Falle das Auftreten zweier stellungsisomerer Nitrokörper, die durch den Schmelzpunkt unterschieden waren und durch fractionierte Krystallisation aus Alkohol getrennt werden konnten. Die $\beta$-Verbindung krystallisierte bei Anwendung von viel Alkohol zuerst aus und zeigte nach wiederholtem Umkrystallisieren den constanten Schmelzpunkt 100 bis $102^{\circ}$. Lange, hellgelbe, durchsichtige Nadeln.

1 Chem. Centralblatt, 1899 , T. 878. 
I. $0.2115 \mathrm{~g}$ im Vacuum über Schwefelsäure getrocknete Substanz gaben $0 \cdot 4238 \mathrm{~g}$ Kohlensäure und $0 \cdot 1030 \mathrm{~g}$ Wasser.

II. $0 \cdot 3154 \mathrm{~g}$ im Vacuum über Schwefelsäure getrocknete Substanz gaben bei $20 \cdot 0^{\circ}$ und $752.5 \mathrm{~mm}$ Druck $20.1 \mathrm{~cm}^{3}$ Stickstoff.

In 100 Theilen:

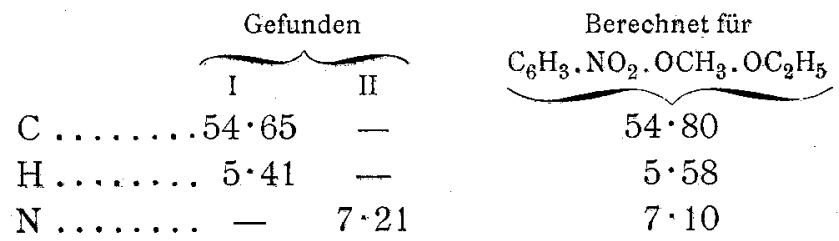

Dieser in Alkohol schwer lösliche Körper entsteht nur in sehr geringer Menge, etwa $10 \%$. Die Hauptmenge $(\alpha$-Verbindung) des gebildeten Nitrokörpers ist in Alkohol leichter löslich und zeigt den constanten Schmelzpunkt 65 bis $67^{\circ}$. Kleine hellgelbe Nadeln.

I. $0 \cdot 2023 \mathrm{~g}$ im Vacuum über Schwefelsäure getrocknete Substanz ergaben $0.4054 \mathrm{~g}$ Kohlensäure und $0.1056 \mathrm{~g}$ Wasser.

II. $0 \cdot 3837 . g$ im Vacuum über Schwefelsäure getrocknete Substanz ergaben bei $18^{\circ}$ und $750.7 \mathrm{~mm}$ Druck $25 \mathrm{~cm}^{3}$ Stickstoff.

In 100 Theilen:
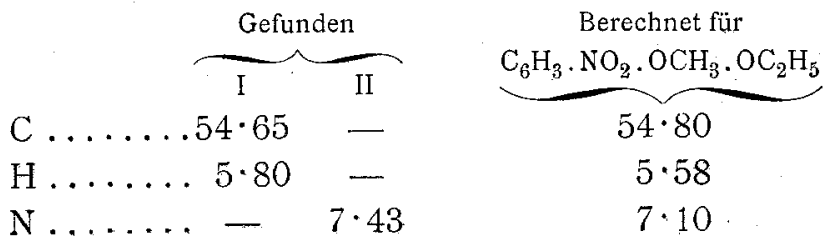

Aus $50 \mathrm{~g}$ Äther wurden $5 \mathrm{~g}$ des höher schmelzenden und $48 \mathrm{~g}$ des niedriger schmelzenden Nitrokörpers gewonnen, d. i. $81 \%$ der theoretischen Ausbeute.

Reduction der Nitroderivate.

Je $30 \mathrm{~g}$ der erwähnten Nitrokörper wurden mit ungefähr $50 \mathrm{~g}$ concentrierter Salzsäure aufgeschlemmt und allmählich $60 \mathrm{~g}$ Zinn zugesetzt unter fortwährendem Umschütte!n und Kühlen. Nach Verlauf von zwei bis drei Stunden ist der anfänglich gelbe Nitrokörper vollständig in eine homogene 
weiße Masse übergegangen. Das Ende der Reduction ist daran kenntlich, dass eine herausgenommene Frobe vollständig in Wasser löslich ist, und somit keinen unveränderten Nitrokörper mehr enthält. Die ganze Masse wird nunmehr in der nöthigen Menge Wasser gelöst, die Flüssigkeit vom überschüssigen Zinn abfiltriert und zur Entfernung der Salzsäure im Vacuum eingedampft. Das zurückbleibende Zinndoppelsalz wird in viel Wasser gelöst und das Zinn durch Schwefelwasserstoff ausgefällt, der Niederschlag abfiltriert und sorgfältig gewaschen, die Flüssigkeit neuerdings im Vacuum bis zur Trockene eingedampft. Das zurückbleibende salzsaure Amidoproduct ist anfänglich weiß, verfärbt sich aber bald bei Luftzutritt. Des. gleichen nimmt die anfänglich farblose wässerige Lösung bald eine dunkelviolette Farbe an. Aus $30 \mathrm{~g}$ Nitrokörper wurden bis zu $25 \mathrm{~g}$ Chlorhydrat erhalten, d. i. $80 \%$ der theoretischen Ausbeute.

Acetylierung der Amidoverbindungen.

Behufs Überführung der Amidokörper in die Acetylderivate wurde das frisch bereitete Chlorhydrat in einem Überschusse von Essigsäureanhydrid unter Erwärmen gelöst, das Anhydrid sodann im Vacuum abdestilliert, bis absolut nichts mehr übergieng, der syrupartige Rückstand keinen Geruch nach Anhydrid mehr zeigte und beim Auskühlen erstarte. Derselbe wurde nun in Benzol unter Erwärmen gelöst, mit wenig Ligroin versetzt und der Krystallisation überlassen. Auf diesem Wege wurden reine Diacetylproducte erhalten, während bei früheren Versuchen, wo Wasser als Lösungsmittel des. Rohproductes verwendet wurde, durch die außerordentlich leicht eintretende Verseifung der Diacetylkörper stets Gemische von Mono- und Diacetylverbindungen entstanden, was zunächst durch sehr unregelmäßige Schmelzpunkte zum Ausdrucke kam. Dank dieser Beobachtung war jedoch gleichzeitig ein Weg gewiesen, um von den leicht darstellbaren Diacetylkörpern zu den verhältnismäßig schwer zugänglichen Monoacetylderivaten durch partielle Rückverseifung zu gelangen. Dies wurde auch thatsächlich durch anhaltendes Kochen der Diacetylproducte des Methyläthyläthers mit Wasser erreicht; beim Diäthylderivate 
gelang es hingegen nur bei Gegenwart von wenig Calciumcarbonat.

\section{Diacetyl- $\alpha$-Amidobrenzcatechinmethyläthyläther.}

Aus Benzol unter Zusatz von Ligroin umkrystallisiert, zeigt diese Verbindung den constanten Schmelzpunkt 117 bis $119^{\circ}$. Die Analysen lieferten folgende Werte:

I. $0.2503 \mathrm{~g}$ im Vacuum über Schwefelsäure getrocknete Substanz gaben $0.5730 \mathrm{~g}$ Kohlensäure und $0.1441 \mathrm{~g}$ Wasser.

II. $0.2009 \mathrm{~g}$ im Vacuum über Schwefelsäure getrocknete Substanz gaben $0.4583 \mathrm{~g}$ Kohlensäure und $0.1184 \mathrm{~g}$ Wasser.

III. $0.2909 \mathrm{~g}$ im Vacuum über Schwefelsäure getrocknete Substanz gaben bei $15^{\circ}$ und $740 \mathrm{~mm}$ Druck $13.7 \mathrm{~cm}^{3}$ Stickstoff.

In 100 Theilen:

\begin{tabular}{|c|c|c|c|}
\hline \multicolumn{3}{|c|}{ Gefunden } & \multirow{2}{*}{$\begin{array}{c}\text { Berechnet für } \\
\mathrm{C}_{6} \mathrm{H}_{3} \cdot \mathrm{OCH}_{3} \cdot \mathrm{OC}_{2} \mathrm{H}_{5} \cdot \mathrm{N}\left(\mathrm{CH}_{3} \cdot \mathrm{CO}\right)_{2} \\
\end{array}$} \\
\hline I & II & III & \\
\hline С....662 443 & $62 \cdot 43$ & - & $62 \cdot 15$ \\
\hline $\mathrm{H} \ldots \ldots 6 \cdot 39$ & $6 \cdot 55$ & - & $6 \cdot 76$ \\
\hline$\ldots \quad-$ & — & $5 \cdot 46$ & $5 \cdot 58$ \\
\hline
\end{tabular}

I. $0 \cdot 2132 \mathrm{~g}$ im Vacuum über Schwefelsäure getrocknete Substanz verbrauchten bei der Acetylbestimmung nach Wenzel $16.6 \mathrm{~cm}^{3} 1 / 10 \mathrm{n}$. Kalilauge.

II. $0 \cdot 2561 \mathrm{~g}$ im Vacuum über Schwefelsäure getrocknete Substanz verbrauchten bei der Acetylbestimmung nach Wenzel $20.6 \mathrm{~cm}^{3} 1 / 10 \mathrm{n}$. Kalilauge.

In 100 Theilen:

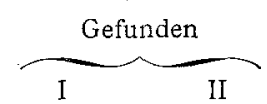

$\mathrm{CH}_{3} \mathrm{CO} \quad \ldots 33 * 48 \quad 34 \cdot 59$
Berechnet für

$\mathrm{C}_{6} \mathrm{H}_{3} \cdot \mathrm{OCH}_{3} \cdot \mathrm{OC}_{2} \mathrm{H}_{5} \cdot \mathrm{N} \cdot\left(\mathrm{CH}_{3} \cdot \mathrm{CO}\right)_{2}$

$34 \cdot 26$

Dieses Diacetylproduct wurde in viel kochendem Wasser gelöst und am Rückflusskühler 24 Stunden gekocht, sodann eingeengt und der Krystallisation überlassen. Kleine weiße Blättchen vom Schmelzpunkte 133 bis $135^{\circ}$.

Die Acetylbestimmung lieferte $24 \cdot 4 \% \mathrm{CH}_{3} \mathrm{CO}$ gegen $20 \cdot 57$ für das Monoacetylderivat.

Hiedurch war erwiesen, dass dem Producte noch Diacetylkörper beigemengt war. Nach weiterem Kochen und wieder- 
holtem Umkrystallisieren aus Wasser wurden schöne, weiße Blättchen vom Schmelzpunkte 136 bis $138^{\circ}$ erhalten.

I. $0.1803 \mathrm{~g}$ im Vacuum über Schwefelsäure getrocknete Substanz verbrauchten bei der. Acetylbestimmung nach Wenzel $8.9 \mathrm{~cm}^{3} 1 / 10 \mathrm{n}$. Kalilauge.

II. $0.1779 \mathrm{~g}$ im Vacuum über Schwefelsäure getrocknete Substanz verbrauchten bei der Acetylbestimmung nach Wenzel $8.7 \mathrm{~cm}^{3} \quad 1 / 10 \mathrm{n}$. Kalilauge.

In 100 Theilen:

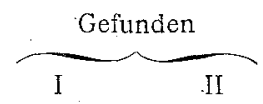

$$
\mathrm{CH}_{3} \mathrm{CO} \ldots 21 \cdot 23 \quad 21 \cdot 03
$$

Berechnet für

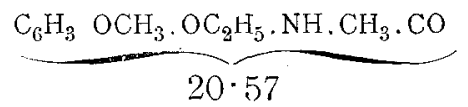

Bei neuerlicher Darstellung wurde eine Spur Calciumcarbonat zugesetzt und auch sechs Stunden gekocht, worauf sich dreimaliges Umkrystallisieren aus Wasser als ausreichend erwies, um den obigen Schmelzpunkt 136 bis $138^{\circ}$ zu erreichen.

Die Analyse dieser Verbindung ergab folgendes Resultat:

I. $0 \cdot 1908 \mathrm{~g}$ im Vacuum über Schwefelsäure getrocknete Substanz ergaben $0.4410 \mathrm{~g}$ Kohlensäure und $0.1219 \mathrm{~g}$ Wasser.

II. $0.4555 \mathrm{~g}$ im Vacuum über Schwefelsäure getrocknete Substanz ergaben bei $20 \cdot 0^{\circ}$ und $747 \cdot 4 \mathrm{~mm}$ Druck $26 \cdot 8 \mathrm{~cm}^{3}$ Stickstoff.

In 100 Theilen:

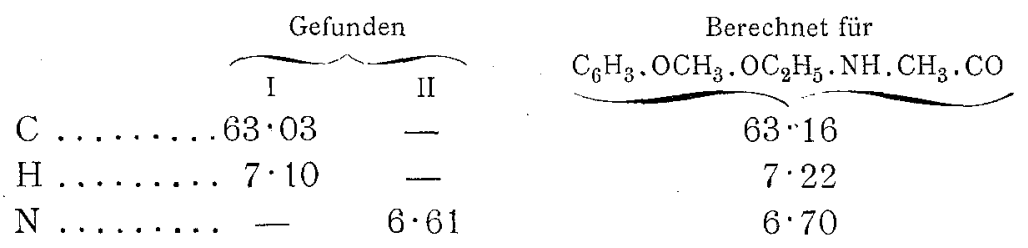

\section{Diacetyl-3-Amidobrenzcatechinmethyläthyläther.}

Der isomere, bei 100 bis $102^{\circ}$ schmelzende Nitrokörper ist ebenfalls in das Amidoproduct und dieses sodann auf die gleiche Weise in das Diacetylproduct übergeführt worden. Nach wiederholtem Umkrystallisieren aus Benzol wurden kleine weiße Krystalle vom Schmelzpunkte 135 bis $137^{\circ}$ erhalten.

I. $0 \cdot 2170 \mathrm{~g}$ im Vacuum über Schwefelsäure getrocknete Substanz gaben $0 \cdot 4934 \mathrm{~g}$ Kohlensäure und $0.1357 \mathrm{~g}$ Wasser. 
II. $0.2315 \mathrm{~g}$ im Vacuum über Schwefelsäure getrocknete Substanz verbrauchten bei der Acetylbestimmung nach Wenzel $18.4 \mathrm{~cm}^{3} 1 / 10 \mathrm{n}$. Kalilauge.

In 100 Theilen:

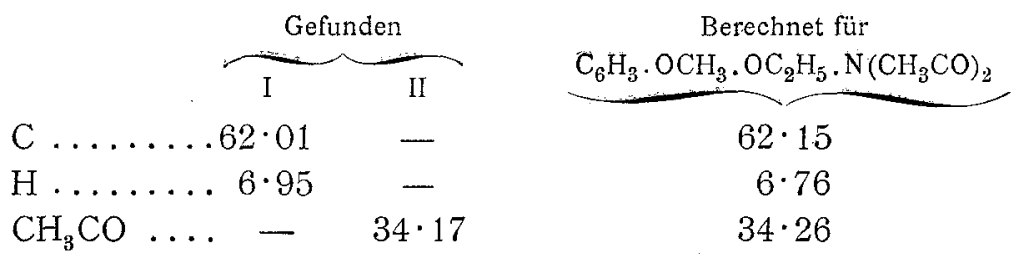

Durch sechsstündiges Kochen mit Wasser unter Zusatz von Calciumcarbonat und nach dreimaligem Umkrystallisieren wurde das Monoacetylproduct in prachtvollen silberglänzenden Blättchen erhalten, und zeigte dasselbe den constanten Schmelzpunkt 142 bis $143^{\circ}$.

\section{Diacetylamidobrenzcatechindiäthyläther.}

In der bereits erwähnten Weise dargestellt, krystallisiert dieser Körper in großen durchsichtigen Blättchen von glimmerartigem Glanze und dem constanten scharfen Schmelzpunkte 120 und $121^{\circ}$.

Die Analysen lieferten folgende Werthe:

I. $0 \cdot 2245 \mathrm{~g}$ im Vacuum über Schwefelsäure getrocknete Substanz gaben $0.5209 \mathrm{~g}$ Kohlensäure und $0.1347 \mathrm{~g}$ Wasser.

II. $0.2056 \mathrm{~g}$ im Vacuum über Schwefelsäure getrocknete Substanz gaben $0.4768 \mathrm{~g}$ Kohlensäure und $0.1343 \mathrm{~g}$ Wasser.

III. $0.2324 \mathrm{~g}$ im Vacuum über Schwefelsäure getrocknete Substanz gaben bei $14^{\circ}$ und $746 \mathrm{~mm}$ Druck $11 \mathrm{~cm}^{3}$ Stickstoff.

In 100 Theilen:

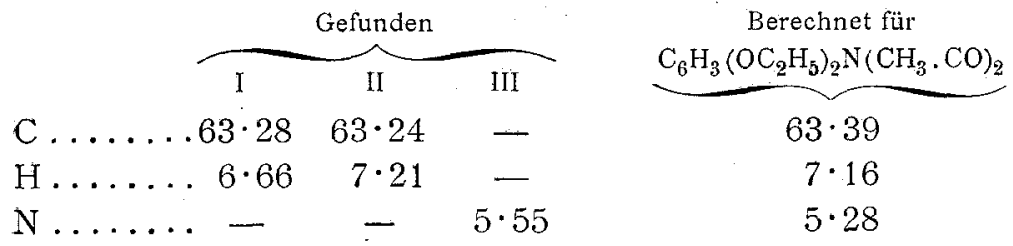

I. $0 \cdot 1937 \mathrm{~g}$ im Vacuum über Schwefelsäure getrocknete Substanz verbrauchten bei der Acetylbestimmung nach Wenze1 $14 \cdot 3 \mathrm{~cm}^{3} \quad 1 / 10 \mathrm{n}$. Kalilauge. 
II. $0.2012 \mathrm{~g}$ im Vacuum über Schwefelsäure getrocknete Substanz verbrauchten bei der Acetylbestimmung nach Wenzel $15 \cdot 2 \mathrm{~cm}^{3} 1 / 10 \mathrm{n}$. Kalilauge.

In 100 Theilen:

\begin{tabular}{|c|c|c|c|}
\hline \multirow{2}{*}{\multicolumn{3}{|c|}{ Gefunden }} & \multirow{2}{*}{$\begin{array}{c}\text { Berechnet für } \\
\mathrm{C}_{6} \mathrm{H}_{3}\left(\mathrm{OC}_{2} \mathrm{H}_{5}\right)_{2} \mathrm{~N}\left(\mathrm{CH}_{3} \mathrm{CO}\right)_{2}\end{array}$} \\
\hline & & & \\
\hline & $31 \cdot 74$ & $32 \cdot 40$ & $32 \cdot 45$ \\
\hline
\end{tabular}

Das Monoacetylderivat erhält man in Form schöner, weißer, durchsichtiger Blättchen vom cōnstanten Schmelzpunkte 125 bis $126^{\circ}$.

Die Analysen lieferten folgende Daten:

I. $0.2553 \mathrm{~g}$ im Vacuum über Schwefelsäure getrocknete Substanz verbrauchten bei der Acetylbestimmung nach Wenzel $11.5 \mathrm{~cm}^{3} 1 / 10 \mathrm{n}$. Kalilauge.

II. $0 \cdot 2505 \mathrm{~g}$ im Vacuum über Schwefelsäure getrocknete Substanz lieferten nach $Z$ e is el $0.5172 \mathrm{~g}$ Jodsilber.

In 100 Theilen:
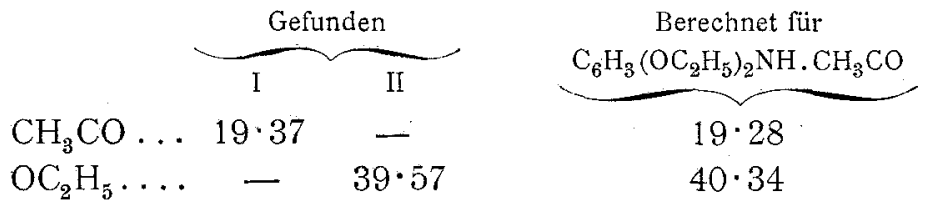

\section{Stellungsnachweis.}

Der Umstand, dass in beiden Fällen die Nitrierung, unter denselben Bedingungen eingeleitet, ganz gleichartig verläuft, gestattet den Schluss, dass die Nitrogruppe bei beiden Äthern an dieselbe Stelle tritt; und die Isomerie beim Methyläthyläther ist wohl nur durch die verschiedene Stellung der Nitrogruppe zum Methoxyl-, respective Äthoxylrest zu erklären. Es wäre daher als mögliche Stellung für den Eintritt der Nitrogruppe nur 3 und 6 oder 4 und 5 anzunehmen, d. h. sie tritt zu den vorhandenen Substituenten entweder in die Ortho- oder in die Metastellung. Die Constitution -der ganzen Körperreihe ist demnach entweder eine vicinale oder eine asymmetrische, und 
der.Stellungsnachweis ist geliefert, wenn es durch Eliminierung der Nitrogruppe und Ersatz derselben durch die Hydroxylgruppe gelingt, zu dem vicinalen (Pyrogallol) oder asymmetrischen (Oxyhydrochinon) Typus des Trioxybenzols zu gelangen. Da vom Pyrogallol und Oxyhydrochinon die Triäthyläther, von ersterem auch der Diäthyläther bekannt sind, so konnte durch Überführung des Nitroproductes in einen dieser Äther bekannter Stellung die Constitution des Körpers selbst aufgeklärt werden. Aus diesem Grunde wurden die nachfolgenden Versuche nur mit dem Nitroderivate des Diäthyläthers ausgeführt.

Da das salzsaure Amidoproduct sich zur Diazotierung als wenig geeignet erwies (schon infolge seiner geringen Haltbarkeit), so wurde dasselbe zunächst in das Sulfat übergeführt. Dabei musste vor allem darauf Bedacht genommen werden, die außerordentlich empfindliche freie Base möglichst vor Oxydation zu schützen; nach zahlreichen Versuchen hat sich der nachfolgende Weg als der sicherste und in Bezug auf Ausbeute beste erwiesen.

$30 \mathrm{~g}$ frisch bereitetes Chlorhydrat wurden in möglichst wenig Wasser gelöst, die Lösung in der Schüttelflasche mit Äther überschichtet, hierauf die zur Freimachung der Base genau berechnete Menge verdünnter Kalilauge langsam unter Umschütteln zugesetzt. Die freie Base fällt in weißen Flocken aus der wässerigen Lösung aus, löst sich aber beim Durchschütteln sofort in Äther, der dann sorgfältig von der wässerigen Flüssigkeit getrennt wird. Zu der ätherischen Lösung der Base wird nun tropfenweise concentrierte Schwefelsäure unter Umschütteln so lange zugesetzt, bis keine Fällung mehr beobachtet wurde. Der schön weiße, voluminöse Niederschlag wird nun sofort von der ätherischen Lauge abgesaugt und mit Äther nachgewaschen. Aus $30 \mathrm{~g}$ Chlorhydrat wurden auf diese Art bis zu $28 \mathrm{~g}$ Sulfat gewonnen, d. i. $73 \%$ der theoretischen Ausbeute.

Die Zersetzung des auf gewöhnliche Weise dargestellten Diazokörpers bereitete nun mannigfache Schwierigkeiten. Die Versuche, die Zersetzung durch einfaches Aufkochen der Lösung mit Wasser, durch Zutropfenlassen zu siedendem Wasser und 
Destillation mit Wasserdampf, schließlich selbst bei Gegenwart von frisch bereitetem Kupferpulver, herbeizuführen, ergaben sämmtlich ein negatives Resultat. Endlich wurde ein Versuch gemacht, das neuestens in der Technik zur Darstellung von Guajacol aus Orthoanisidin verwendete Kalle'sche Patentverfahren, ${ }^{1}$ wonach trockenes Glaubersalz und concentrierte (2:1) Schwefelsäure bei 135 bis $145^{\circ}$ zugegen sind, auf den vorliegenden Fall anzuwenden.

$9 \mathrm{~g}$ Sulfat wurden in $26 \mathrm{~cm}^{2}$ Wasser gelöst, unter Zusatz von $9 g 50$-procentiger Schwefelsäure, in Eis gekühlt und langsam mit einer Lösung von $2 \cdot 2 \mathrm{~g}$ Natriumnitrit in $6 \mathrm{~cm}^{3}$ Wasser diazotiert und 3 bis 4 Stunden in der Kälte stehen gelassen.

Dann wurde die Lösung durch einen Tropftrichter langsam in eine große Retorte eintropfen gelassen, in der ein Gemisch von $100 \mathrm{~g}$ entwässertem Glaubersalz und $135 \mathrm{~g}$ concentrierter Schwefelsäure mit $75 \mathrm{~g}$ Wasser sich befand. Diese Retorte wurde im Ölbade erwärmt, bis innen eine Temperatur von 135 bis $145^{\circ}$ herrschte, und dann Wasserdampf durchgeleitet. So wird jeder einfallende Tropfen der Diazolösung sofort zersetzt und der gebildete Phenoläther von den Wasserdämpfen übergeführt. In die Vorlage war ein gelb gefärbtes Öl übergegangen, allerdings in sehr geringer Menge, das dann in Äther aufgenommen wurde, und nach dem Abdunsten desselben langsam zu schönen, kleinen, weißen Nadeln erstarrte. Zur Reinigung wurde dasselbe im Vacuum destilliert $\left(200\right.$ bis $220^{\circ}$ bei $15 \mathrm{~mm}$ Druck) und zeigten die Krystalle nach dem Umkrystallisieren sowohl aus Benzol, als auch aus Alkohol den constanten Schmelzpunkt 65 bis $67^{\circ}$.

Die Analysen lieferten folgende Werte:

I. $0 \cdot 1461 \mathrm{~g}$ im Vacuum über Schwefelsäure und Kalk getrocknete Substanz ergaben $0.3538 \mathrm{~g}$ Kohlensäure und $0.1062 \mathrm{~g}$ Wasser.

II. $0 \cdot 1586 \mathrm{~g}$ im Vacuum über Schwefelsäure und Kalk getrocknete Substanz ergaben nach $Z$ eise $10 \cdot 4071 \mathrm{~g}$ Jodsilber.

1. Kalle \& Co., Biebrich am Rhein. Friedländer, Theerfarbenfabrication, S. 124. 
In 100 Theilen:

$\begin{array}{lcc}\text { I }_{\text {I }}^{\text {Gefunden }} & \text { II } & \underbrace{\mathrm{C}_{6} \mathrm{H}_{3}\left(\mathrm{OC}_{2} \mathrm{H}_{5}\right)_{2} \mathrm{OH}}_{66.04} \\ \mathrm{H} \ldots \ldots . & -\end{array}$

Dadurch war der Körper als Trioxybenzoldiäthyläther ausreichend identificiert und konnte derselbe sonach nur Diäthylpyrogallol- oder Diäthyloxyhydrochinonäther sein. Der erstere ist aus den Arbeiten von Benedict ${ }^{1}$ und $\mathrm{Hofmann}{ }^{2}$ bekannt, und hat Hofmann für denselben durch die Cedriretreaction nachgewiesen, dass er die Äthoxylgruppen in vicinaler Stellung enthält. Dieser Äther wäre also nach Hofmann an dem Schmelzpunkte $\left(79^{\circ}\right)$ und an der auch mit geringen Mengen ausführbaren Coerulignonreaction leicht kenntlich. Es wurde versucht, diese letztere genau nach den Angaben von Hofman $n$ ausszuführen, jedoch mit negativem Resultate. So wäre aus dem Ausbleiben der Kriterien des Pyrogalloldiäthers (Schmelzpunkt und Cedriretreaction) mit großer Wahrscheinlichkeit anzunehmen, dass man es in diesem Falle mit dem bisher noch nicht dargestellten Oxyhydrochinondiäther $z u$ thun habe. Da sowohl der Pyrogalloltriäther (Hofmann), als auch der Oxyhydrochinontriäther (Will, Pukall; ${ }^{3}$ Will, Albrecht ${ }^{4}$ ) bekannt und durch den Schmelzpunkt unterschieden sind, wurde versucht, aus dem vorliegenden Diäther den Triäther zu gewinnen.

Die zur Verfügung gestandene Substanzmenge (ungefähr 1 g) wurde in Alkohol gelöst, mit einem Überschusse von alkoholischem Kali versetzt und durch sechs Stunden am Rückflusse mit einem Überschusse von Jodäthyl digeriert. Der Alkohol wurde abdestilliert, der Rückstand in Wasser aufgenommen und mit Äther extrahiert, die ätherische Lösung bis
1 B., IX, 125.
2 B., XI, 798.
3 B., XX, 1133.
4 B., XVII, 2108. 
zur Entfernung von allem kalilöslichen Diäther mit Kali geschüttelt und der Äther abgedunstet. Das zurückgebliebene Öl erstarrte, und die Krystallmasse zeigte den Schmelzpunkt 32 bis $33^{\circ}$. Da nun der Oxyhydrochinontriäther den Schmelzpunkt $34^{\circ}$ besitzt (Wi11), so wäre wohl die Identität festgestellt, wenn nicht das geringe Intervall gegenüber dem Schmelzpunkte des Pyrogalloltriäthers $\left(39^{\circ}\right.$, Hofmann) einen Zweifel als berechtigt erscheinen ließe.

Um dieses Bedenken zu entkräften, wurde versucht, von dem vorliegenden Triäther zu dem Bromproducte zu gelangen, welches neuestens im hiesigen Laboratorium von Herrn Bre $z$ in $\mathrm{a}^{1}$ dargestellt wurde. Dasselbe wurde durch Bromieren des Oxyhydrochinontriäthers in ätherischer Lösung gewonnen und besaß den Schmelzpunkt 50 bis $52^{\circ}$ und die Zusammensetzung eines Monobromderivates.

$\mathrm{Zu}$ diesem Behufe wurde genau nach den Angaion Brezinas die vorhandene Menge Triäther in Äther gelöst und tropfenweise die für Einführung von einem Bromatom ausreichende Menge Brom zugesetzt, ein eventueller Überschuss durch Schütteln mit schwefliger Säure entfernt, der Äther mit Wasser gewaschen und verdunstet. Das zurückgebliebene Öl erstarrte zu einer Krystallmasse, die sich aus Alkohol gut umkrystallisieren ließ und thatsächlich den von Brezina gefundenen Schmelzpunkt von 51 bis $52^{\circ}$ besaß.

$0.1877 \mathrm{~g}$ im Vacuum ïber Kalk und Schwefelsäure getrocknete Substanz lieferten nach $Z$ eise $10 \cdot 4559 \mathrm{~g}$ Jodsilber.

In 100 Theilen:

$$
\mathrm{OC}_{2} \mathrm{H}_{5} \ldots \underbrace{\text { Gefunden }}_{46 \cdot 66} \quad \underbrace{\begin{array}{c}
\text { Berechnet für } \\
\mathrm{C}_{6} \mathrm{H}_{2} \cdot \mathrm{Br} \cdot\left(\mathrm{OC}_{2} \mathrm{H}_{5}\right)_{3}
\end{array}}_{46 \cdot 71}
$$

Hiedurch ist bewiesen, dass die ganze Körperreihe nach dem asymmetrischen Typus constituiert ist, und dass die Nitrogruppe zu den vorhandenen Substituenten in die Metastellung

1 Diese Beobachtung soll nächstens in einer größeren Arbeit publiciert werden. 
getreten ist. Es ergibt sich demnach für den Nitrokörper des Diäthyläthers folgendes Schema:

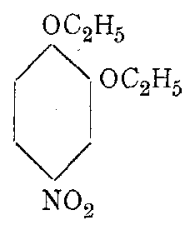

und für die beiden isomeren Nitrokörper des Methyläthyläthers:<smiles>CCOC1CCN([N+](=O)[O-])CCO1</smiles><smiles>CCCCOC1CCC([N+](=O)[O-])CC1</smiles>

Zum Schlusse sei es mir noch gestattet, Herrn Prof. Herzig für all seine Mühe, Freundlichkeit und Umsicht herzlichst zu danken, mit der er diese Arbeit geleitet hat. 